\title{
Swallowing-aspiration functional assessment in patients undergoing supracricoid subtotal laryngectomy with cricohyoidoepiglottopexy
}

\author{
José Francisco Gallegos-Hernández, María Elba Díaz-Guzmán, Pablo Antonio Pichardo-Romero, \\ Oscar Omar Mateos-Aguilar, José Alberto Abrego and Alma Lilia Ortiz-Maldonado \\ Oncology Hospital, Centro Médico Nacional Siglo XXI, Instituto Mexicano del Seguro Social, Ciudad de México, Mexico
}

\begin{abstract}
Introduction: Supracricoid subtotal laryngectomy associated with cricohyoidoepiglottopexy is the most efficient conservative surgical technique to preserve laryngeal functions in patients with locally advanced carcinoma of the larynx. The most feared complication of this intervention is aspiration at the moment of swallowing and secondary pneumonia; there are several ways to evaluate aspiration and its degree. We present a novel form to identify even small amounts of tracheobronchial aspiration. Method: Patients undergoing subtotal laryngectomy and cricohyoidoepiglottopexy due to laryngeal cancer were included; all of them were postoperatively assessed with radioactive-swallow. Based on the symptoms and the scintigraphy result, prolonging the feeding time by nasogastric tube was decided. Results: Thirty-seven patients were included; 4 of them had received radiotherapy; aspiration rate was $29.7 \% ; 50 \%$ of patients who had received radiation had aspiration; $18 \%$ of subjects with tracheobronchial aspiration required prolongation of feeding time by nasogastric tube, none required total laryngectomy for aspiration that hindered swallowing. Conclusions: Postoperative evaluation of patients undergoing subtotal laryngectomy with radioactive-swallow, allows to identify even minimal amounts of fluid aspirated into the bronchial tree and to plan the moment for swallowing to be started.
\end{abstract}

KEY WORDS: Cricohyoidoepiglottopexy. Laryngectomy. Subtotal laryngectomy. Partial laryngectomy. Swallowing. Laryngeal cancer.

\section{Introduction}

The partial laryngectomy procedure known as subtotal-supracricoid laryngectomy (SSCL) was introduced in Europe between the decades of 1950 and $1970 .^{1,2}$ It was designed as a previous step to total laryngectomy to preserve natural airway ventilation and phonation (which is not possible with total laryngectomy) and allow the patient to swallow without tracheobronchial aspiration, in addition to offering adequate oncological control. ${ }^{1}$

Currently, SSCL plays an important role in the treatment of patients with squamous cell carcinoma of the larynx at locally advanced stages who are not candidates for non-surgical conservative treatment either with radiotherapy alone or with concomitant chemo-radiotherapy, ${ }^{3}$ as well as in some patients with recurrence after radiotherapy. However, this procedure is not without complications, with the most common being bronchial aspiration when the tracheotomy cannula is removed and swallowing is initiated. Significant aspiration is an indication for maintaining the tracheotomy cannula to avoid the risk of aspiration pneumonia or to continue nasogastric tube feeding for longer; persistent bronchoaspiration despite swallowing rehabilitation is an absolute indication for converting the conservative procedure into total laryngectomy. ${ }^{4,5}$

Bronchoaspiration should be timely diagnosed, since it may not be clinically evident and manifest itself late with pneumonic foci, which seriously compromises
Correspondence: José Francisco Gallegos-Hernández E-mail: jfgalh61@gmail.com
Date of reception: 01-12-2017

Date of acceptance: 10-09-2018

DOI: 10.24875/GMM.M19000205
Gac Med Mex. 2018;154:546-549

Contents available at PubMed www.gacetamedicademexico.com 
postoperative evolution, deteriorates patient health status and is life threatening.

Aspiration in patients undergoing subtotal laryngectomy is important: 30 to $40 \%$ can experience it at varying degrees; it is more common in patients older than 65 years, in whom surgery is performed in the form of rescue after radiotherapy and in patients in whom one of the arytenoid cartilages function has been compromised, either by resection or iatrogenic injury of the inferior laryngeal nerve. ${ }^{4,6,7}$

The usual ways to identify if the patient has bronchial aspiration when swallowing are clinical evaluation (interrogation aimed at knowing if there is cough when swallowing), videoendoscopy and videofluorescence. However, with these methods, mild to moderate amounts of aspirate fluids can go unnoticed and have a late impact on patient ventilatory status.

The purpose of the present study is to describe a novel technique to assess aspiration in patients undergoing subtotal supracricoid laryngectomy, which allows identifying minimal amounts of fluid aspiration into the bronchial tree: radioactive-swallow, which allows the planning of tracheotomy management with greater objectivity.

\section{Method}

Patients with squamous cell carcinoma of the larynx who consecutively underwent surgical conservation of the larynx with SSCL and reconstruction with cricohyoidoepiglottopexia (CHEP) were evaluated.

Removal of the nasogastric tube and decannulation were carried out consecutively and according to clinical evaluation. One week after decannulation, the swallow test with radioactive material and scintigraphy was carried out to detect aspirated fluid and decide if feeding tube replacement and rehabilitation was required; if there was no important aspiration, continuing without tube and with rehabilitation exercises was decided.

The surgical technique was similar in all patients: both arytenoid cartilages were preserved and the indications to carry out the procedure were those usually reported; in all patients, measures were taken during the transoperative period to minimize aspiration (placement of endolaryngeal stitches, stitches for corniculated cartilages fixation and closing the pharyngeal gap was avoided in order for not to favor piriform sinus rotation and thereby aspiration).

For the radioactive swallow technique, an acquisition protocol was followed; $1 \mathrm{mCi}$ of ${ }^{99 \mathrm{~m}} \mathrm{Tc}$ colloidal

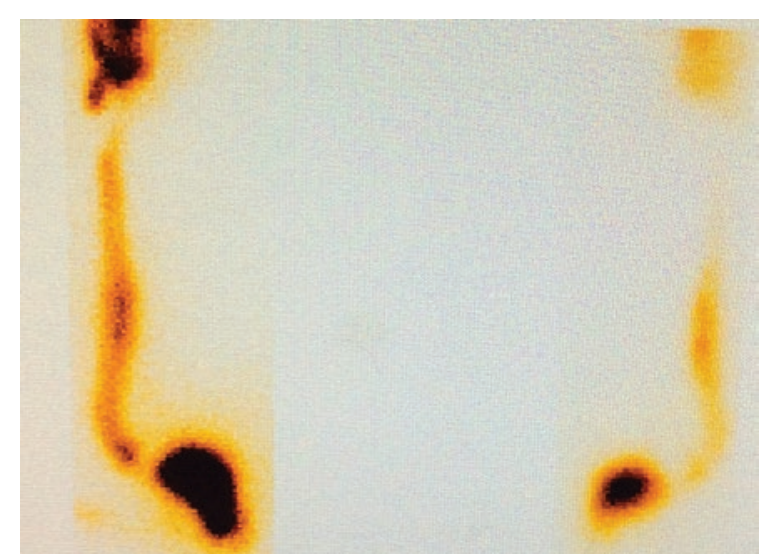

Figure 1. Radioactive swallow showing radiopharmaceutical adequate passage towards the esophagus without bronchial aspiration, which occurred in $70.3 \%$ of patients; gastrostomy tube and gastrostomy cannula removal can be carried out without bronchoaspiration risk.

sulfide in $1 \mathrm{~mL}$ volume was orally administered, a Phillips Precedence T16 gamma camera was used, as well as a low energy collimator for general purposes, with a $20 \%$ window, centered on $140 \mathrm{KeV}$ photope.

A $64 \times 564$ dynamic study and $256 \times 256$ static images were carried out. The images taken at the dynamic phase were 30 of 2 seconds, and at the static phase, an immediate image of 2 to 3 minutes' duration and another at two hours, of 5 minutes' duration. The projections were anterior and posterior, and the field of vision covered the maxillary region up to the epigastrium.

With the patient standing in front of the gamma camera detector, $1 \mathrm{~mL}$ of the radiopharmaceutical was orally administered, with the dynamic study being started for 60 seconds; subsequently the patient was to swallow $5 \mathrm{~mL}$ of water and immediate static images were taken and at two hours the delayed ones.

Qualitative (visual) assessment was carried out in order to identify the passage of the radiopharmaceutical to the airway and quantitative assessment was performed by tracing areas of interest (Fig. 1).

Regardless of the clinical status, patients who had aspiration underwent intensive speech and swallowing rehabilitation if they had no symptoms or had tracheotomy cannula and nasogastric tube placement, in addition to rehabilitation, if there was clinical evidence of aspiration.

\section{Results}

Thirty-seven patients undergoing SSCL and CHEP due to squamous cell carcinoma of the larynx were included; in 33, the surgical procedure was carried out 
as primary intervention, in three, as rescue after radiotherapy, and in one, after bio-radiotherapy.

The radioactive swallow showed bronchial aspiration in $11 / 37$ patients $(29.7 \%$ ) and did not show radioactive material in the tracheobronchial region in $70.3 \%$. Out of them, $8 / 33$ of those who had not received previous treatment $(24 \%)$ had aspiration and $2 / 4(50 \%)$ of those who had received treatment (radiotherapy) had bronchial aspiration. The patient with a history of bio-radiotherapy had the highest amount of aspiration and required more nasogastric tube time.

Patients undergoing the procedure as initial intervention had an aspiration rate of $27.7 \%$, whereas in those operated in the form of rescue, the aspiration rate was $50 \%$.

Among the patients who showed aspiration (11), $3(27 \%)$ had important clinical symptomatology that required feeding tube: in two for 10 days more and in another one for three more weeks, after which the swallow showed minimal aspiration and swallowing was adequately tolerated.

Patients who in the scintigraphy showed aspiration limited to the trachea or minimal aspiration to one of the bronchi tolerated decannulation and returned to a normal diet in an average of two weeks after decannulation and nasogastric tube withdrawal.

All 37 patients were finally decanulated and had the nasogastric tube removed; no one showed pneumonic foci during their treatment or required SSCL conversion to total laryngectomy; mean follow-up time is two years and no one has shown aspiration complications.

\section{Discussion}

SSCL with CHEP is a suitable alternative to preserve laryngeal functions in patients with locally advanced laryngeal cancer who are not candidates for radiotherapy or chemo-radiotherapy or as a rescue procedure due recurrence to radiotherapy or chemo-radiotherapy. ${ }^{3,8,9} \mathrm{It}$ allows achieving adequate oncological control and acceptable functionality of the larynx; the most important complication is food aspiration, which has been associated with older age, ${ }^{10}$ resection of an arytenoid and endolaryngeal granulomas formation. An adequate surgical technique enables to minimize the aspiration rate, which in many cases is not clinically evident. ${ }^{11}$

Postoperative assessment of swallowing is fundamental in order to identify tracheobronchial aspiration and initiate measures to reduce the possibility of complications due to pneumonia; ${ }^{12}$ radioactive swallow with scintigraphy allows to identify the amount of aspiration and decide if the patient is a candidate for rehabilitation exercises, repositioning of the nasogastric tube or tracheotomy cannula and eventually for conversion of the procedure to total laryngectomy.

In the present series, aspiration was identified in almost $30 \%$ of patients, in $82 \%$ of them the amount of aspirated fluid evaluated with post-radioactive swallow scintigraphy was limited to the trachea and to only one of the bronchi in minimal quantity, which allowed swallowing resumption without risk of major aspiration; these patients did not require tracheotomy cannula or nasogastric tube placement and minimal aspiration was resolved with postoperative swallowing therapy; $18 \%$ of patients required more nasogastric tube feeding time and longer tracheotomy time. No one required conversion to total laryngectomy or experienced pulmonary complications during the follow-up; all returned to a normal diet.

In the present series, although the number of patients in whom SSCL was carried out as rescue (relapse after radiotherapy) was small, the aspiration rate was higher in them (50\%), which was probably associated with cricoarytenoid joint dysfunction due to post-radiotherapy morbidity.

Failure to assess swallowing in the postoperative period of conservative laryngeal surgery makes for the removal of the nasogastric tube to be decided based on clinical symptomatology, ${ }^{13}$ which favors longer tube nutrition time and rehabilitation delay. ${ }^{7,13}$

The radioactive swallow, commonly used in children for the assessment of bronchoaspiration due to a number of causes,$^{14}$ is useful to identify even the smallest amount of fluid aspirated to the tracheobronchial tree; based on the result, the ideal moment for tracheostomy tube removal and swallowing resumption is decided, which minimizes the possibility of respiratory complications.

\section{References}

1. Majer EH, Rieder W. Modification of laryngectomy with preservation of the air passages. Arch Ohren Nasen Kehlkopfheilkd. 1958;173:442-446.

2. Piquet JJ. Functional laryngectomy (cricohyoidopexy). Clin Otolaryngol Allied Sci. 1976;1:7-16.

3. Gallegos-Hernández JF, Cruz-Esquivel I, Ortiz-Maldonado AL Minauro-Muñoz GG, Arias-Ceballos H, Pichardo-Romero P. Laryngeal conservative surgery in patients candidates for combined treatment with chemoradiotherapy. Cir Cir. 2016;84:96-101.

4. Leone CA, Capasso P, Topazio D, Russo G. Supracricoid laryngectomy for recurrent laryngeal cancer after chemoradiotherapy: a systematic review and meta-analysis. Acta Otorhinolaryngol Ital. 2016;36:439-449. 
5. Zietek E, Jaworowska E, Tarnowoska C, Jach $\mathrm{K}$ Functional results after supracricoid reconstructive laryngectomy with cricohyoidopexy (chp) and cricohioidoepiglotopexy (chep). Otolaryngol Pol 1992;46:457-462.

6. Allegra E, Franco T, Traspasso S, Domanico R, La-Boria A, Garozzo A. Modified supracricoid laryngectomy: oncological and functional outcomes in the elderly. 2012; 7:475-480.

7. Clayburgh DR, Graville DJ, Palmer AD, Schindler JS. Factors associated with supracricoid laryngectomy functional outcomes. Head Neck. 2013;35:1397-1403.

8. Manelli G, Lazio M, Luparello P, Gallo O. Conservative treatment for advanced T3-4 laryngeal cancer: meta-analysis of key oncological outcomes. Eur Arch Otorhinolaryngol. 2018;275:27-38.

9. Luna-Ortiz K, Pashe P, Tamez-Velarde M, Villavicencio-Valencia V. Spracricoid partial laryngectomy with cricohyoidoepiglottopexy in patients with radiation therapy failure. World J Surg Oncol. 2009;7:101.
10. Laccourreye O, Brasnu D, Périé D, Muscatello L, Ménard M, Weinstein G. Supracricoid partial laryngectomies in the elderly: mortality, complications and functional outcome. Laryngoscope. 1998;108:237-242.

11. Topaloglu I, Köprücü G, Bal M. Analysis of swallowing function alter supracricoid laryngectomy with cricohyoidopexy. Otoalryngol Head Neck Surg. 2012;146:412-418

12. Yücetürk AV, Tarhan S, Günhan K, Pabudçu Y. Videofluorscopic evaluation of the swallowing function after supracricoid laryngectomy. Eur Arch Otorhinolaryngol. 2005;262:198-203.

13. De Vincentiis MI, Calcagno P, Di-Cello P, Mastronicola R, Simnelli M, Ruoppolo G, et al. Transit time of swallowing after subtotal laryngectomy. Rev Laryngol Otol Rhinol (Bord). 2004;125:223-227.

14. Heyman $\mathrm{S}$. The radionuclide salivagram for detectin the pulmonary aspiration of saliva in an infant. Pediatr Radiol. 1989;19:208-209. 